\title{
Spontaneous emergence of angular momentum Josephson oscillations \\ in coupled annular Bose-Einstein condensates
}

\author{
Igor Lesanovsky ${ }^{1, *}$ and Wolf von Klitzing ${ }^{1, t}$ \\ ${ }^{1}$ Institute of Electronic Structure and Laser, \\ Foundation for Research and Technology - Hellas, \\ P.O. Box 1527, GR-71110 Heraklion, Greece
}

(Dated: September 13, 2018)

\begin{abstract}
We investigate the nonlinear dynamics of two coupled annular Bose-Einstein condensates (BECs). For certain values of the coupling strength the nonrotating ground state is unstable with respect to fluctuations in the higher angular momentum modes. The two branched Bogoliubov spectrum exhibits distinct regions of instability enabling one to selectively occupy certain angular momentum modes. For sufficiently long evolution times angular momentum Josephson oscillations spontaneously appear, breaking the initial chiral symmetry of the BECs.
\end{abstract}

PACS numbers: 03.75.Lm, 03.75.Mn, 03.75.Kk 
One of the most famous paradigms of quantum physics is the existence of Josephson oscillations. They were first predicted for superconductors separated by an insulating layer [1]. Later they have been observed in superfluid ${ }^{3} \mathrm{He}$ [2] and gaseous BECs [3, 4]. Next to the oscillations of charge and/or particles between two modes these systems can exhibit highly nonlinear dynamics with sometimes surprising behavior. In this letter we study onedimensional (1D) BECs confined in two ring-shaped traps which are sufficiently close to each other to allow tunneling through the barrier between them. We demonstrate that the stationary state, in which only the zero angular mode is occupied by the BECs, becomes unstable for certain values of the coupling strength. This allows for a selective occupation of specific angular momentum modes. For short propagation times the angular momentum in each ring is conserved. For longer interaction times, however, angular momentum Josephson oscillations appear. This novel type of Josephson oscillations spontaneously breaks the initial chiral symmetry of the individual BECs.

In the mean-field description the evolution of a dilute gas of identical interacting Bosons under the influence of the trapping potential $V(\mathbf{r})$ is governed by the Gross-Pitaevskii equation (GPE), which in cylindrical coordinates reads

$$
i \hbar \partial_{t} \Psi=\left[\frac{\hbar^{2}}{2 M}\left(-\partial_{\rho}^{2}-\partial_{z}^{2}+\frac{L_{z}^{2}}{\hbar^{2} \rho^{2}}\right)+V(\mathbf{r})\right] \Psi+g|\Psi|^{2} \Psi
$$

where $M$ is the atomic mass, $g$ the nonlinear coupling constant, $\Psi=\Psi(\mathbf{r})$ the bosonic mean field and $L_{z}=-\hbar^{2} \partial_{\phi}^{2}$ the $z$-component of the angular momentum operator. The system consist of two BECs in parallel ring-shaped traps encircling the $z$-axis. The positions of the upper and lower ring are $\pm z_{0}$, respectively. Correspondingly, the trapping potential takes the form $V(\mathbf{r})=V_{\rho}\left(\rho-\rho_{0}\right)+V_{z}\left(z, z_{0}\right)$. The first term provides harmonic radial confinement centered at $\rho=\rho_{0}$, and $V_{z}\left(z, z_{0}\right)$ creates a symmetric double well potential with its minima at $z= \pm z_{0}$. Both BECs reside in the radial ground state $\Psi_{\rho}(\rho)$ of $V_{\rho}\left(\rho-\rho_{0}\right)$. Vertically they occupy the harmonic ground states $\Phi\left(z \pm z_{0}\right)$ which are localized in the upper and the lower well of $V_{z}\left(z, z_{0}\right)$, respectively. The total wave function of the system can then be written as $\Psi(\mathbf{r})=\Psi_{\rho}(\rho)\left[\Phi\left(z-z_{0}\right) \chi_{u}(\phi)+\Phi\left(z+z_{0}\right) \chi_{d}(\phi)\right]$ where the indices $u$ and $d$ refer to the upper and lower ring. After inserting $\Psi(\mathbf{r})$ into the GPE we obtain the two coupled equations

$$
i \partial_{\tau} \chi_{u / d}=-\partial_{\phi}^{2} \chi_{u / d}+\kappa \chi_{d / u}+\gamma\left|\chi_{u / d}\right|^{2} \chi_{u / d}
$$


Here we have introduced the scaled time $\tau=\frac{\hbar}{2 M R^{2}} t$, the coupling $\kappa=$ $-R^{2} \int d z \Phi\left(z+z_{0}\right)\left[\partial_{z}^{2}-\frac{2 M}{\hbar^{2}} V(z)\right] \Phi\left(z-z_{0}\right)$ and the interatomic interaction parameter $\gamma=\frac{2 M R^{2} g_{1 D}}{\hbar^{2}} \int d z \Phi^{4}(z)$ with $R^{-2}=\int d \rho \rho^{-2}\left|\Psi_{\rho}(\rho)\right|^{2}$ [15]. We assume that the external confinement allows an effective 1D treatment of the BECs. The atom-atom interaction can then be described by the $1 \mathrm{D}$ coupling constant $g_{1 D}=\frac{2 \hbar^{2}}{M} \frac{a}{a_{\rho}^{2}}[6]$. Here $a$ is the threedimensional s-wave scattering length and $a_{\rho}$ the harmonic oscillator length of the radial ground state. Equations, similar to eq.(2) arise in the context of two coupled elongated condensates [7]. Ring traps additionally allow the existence of stationary currents.

In the angular momentum mode representation the azimuthal wave function of the individual BECs can be written according to $\chi_{u / d}=(2 \pi)^{-1 / 2} \exp \left(i \theta_{u / d}\right) \sum_{m} \alpha_{m}^{u / d} \exp (i m \phi)$ with $\theta_{u / d}$ being the phase of the wave function in the respective annulus. The coefficients $\alpha_{m}^{u / d}$ are normalized such that $\left|\alpha_{m}^{u / d}\right|^{2}=N_{m}^{u / d}$ corresponds to the number of particles residing in the $m$-th angular momentum mode. Hence $\int d \phi\left|\chi_{u / d}\right|^{2}=N^{u / d}$ corresponds to the total number of particles in each of the two annuli. Inserting the above expression for $\chi_{u / d}$ into eq.(2) we find the system of coupled equations

$$
i \partial_{\tau} \alpha_{m}^{u / d}=m^{2} \alpha_{m}^{u / d}+\kappa_{u / d} \alpha_{m}^{d / u}+\frac{\gamma}{2 \pi} \sum_{n n^{\prime}} \alpha_{n}^{u / d} \alpha_{n^{\prime}}^{* u / d} \alpha_{m}^{u / d}
$$

with $\kappa_{u / d}=\kappa_{d / u}^{*}=\kappa e^{i\left(\theta_{d}-\theta_{u}\right)}$. We now seek a stationary solution of this system for which in both of the annuli solely the $m=0$ mode is occupied. This exists only for equal number of particles, i.e. $N_{0}^{u}=N_{0}^{d}=N_{0}$, and equal coupling $\kappa_{u}=\kappa_{d}=\kappa$. We then find the two solutions

$$
\alpha_{0}^{u}= \pm \alpha_{0}^{d}=\sqrt{N_{0}} e^{i(\varepsilon \pm \kappa) \tau+i \theta}, \alpha_{m \neq 0}^{d / u}=0
$$

with some arbitrary phase $\theta$ and $\varepsilon=\frac{\gamma N_{0}}{2 \pi}$ being the nonlinear energy due to the interatomic interaction. Hence the total two-dimensional wave function becomes either a symmetric or an anti-symmetric superposition of the axial ground states of the two annuli:

$$
\Psi_{ \pm}(\mathbf{r})=\sqrt{N_{0}} e^{i \mu_{ \pm} \tau+i \theta} \Psi_{\rho}(\rho)\left[\Phi\left(z+z_{0}\right) \pm \Phi\left(z-z_{0}\right)\right]
$$

with $\mu_{ \pm}=\varepsilon \pm \kappa$ being the chemical potential.

In order to investigate the stability of these states with respect to fluctuations in modes with $m \neq 0$ we make the ansatz $\alpha_{m \neq 0}^{u / d}=e^{i \mu_{ \pm} \tau}\left[u_{m}^{u / d} e^{-i \omega \tau}+v_{m}^{* u / d} e^{i \omega \tau}\right]$. Inserting this together 
with eq.(44) into the eq.(3) yields after linearization in the $u_{m}$ and $v_{m}$ the eigenvalue equation

$$
\begin{aligned}
\omega u_{m}^{u / d} & =\left(m^{2}+\mu_{ \pm}\right) u_{m}^{u / d}+\varepsilon e^{i 2 \theta} v_{-m}^{u / d}+\kappa u_{m}^{d / u} \\
-\omega v_{-m}^{u / d} & =\left(m^{2}+\mu_{ \pm}\right) v_{-m}^{u / d}+\varepsilon e^{-i 2 \theta} u_{m}^{u / d}+\kappa v_{-m}^{d / u}
\end{aligned}
$$

We then find the excitation spectrum consisting of the two branches

$$
\omega_{ \pm}=\sqrt{\left(m^{2}+\varepsilon-\kappa \pm \kappa\right)^{2}-\varepsilon^{2}} .
$$

This result is obtained for either chemical potential $\mu_{ \pm}$. The branch $\omega_{+}$corresponds to the well-known Bogoliubov spectrum [8] of a uniform BEC but with integer $m$. In this letter we consider only repulsive interatomic interaction, i.e. $\varepsilon \geq 0$. Thus $\omega_{+}$is always a real number and the coupled condensates are stable against fluctuations in this excitation branch. Note that $\omega_{+}$is independent of the coupling strength $\kappa$. The $\omega_{-}$branch on the other hand depends on $\kappa$ and result from the interaction among the coupled condensates. Its frequencies are either real or imaginary but never complex. For zero coupling $\omega_{-}$is identical with $\omega_{+}$. Figure 1 depicts for two values of the nonlinear energy $(\varepsilon=1.0$ and $\varepsilon=2.0)$

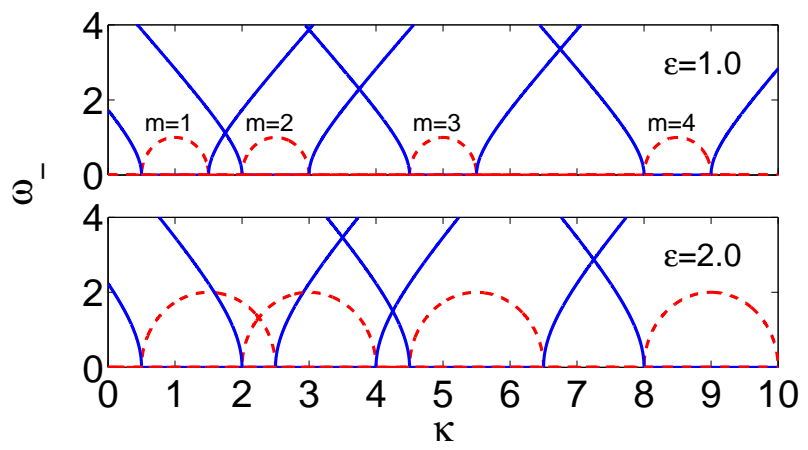

FIG. 1: (Color online) Branch $\omega_{-}$of the Bogoliubov spectrum of the coupled condensates plotted against the coupling strength $\kappa$ for two values of the nonlinear energy $\varepsilon$ (blue/solid: real part, red/dashed: imaginary part). In panel a the instable regions are labelled with the respective $m$ number. Due to the symmetry of eq.(5) the spectrum is symmetric with respect to a sign change of $m$.

the spectrum $\omega_{-}$as a function of the coupling $\kappa$. The blue/solid curves represent the real part and the red/dashed curves the imaginary part of $\omega_{-}$. First, let us consider relatively low energies $(\varepsilon<2)$ (fig. 1a). Starting from $\kappa=0$, we notice that $\omega_{-}$is positive and real, 
and that it decreases monotonously to $\omega_{-}=0$ at $\kappa=1 / 2$. After this, one enters a region where $\omega_{-}$is imaginary with $\operatorname{Im}\left(\omega_{-}\right)>0$. The system is then unstable under fluctuations in the $m= \pm 1$ modes which grow at a rate of $\Gamma=2 \operatorname{Im}\left(\omega_{-}\right)$[7, 9]. As $\kappa$ increases regions of stability and instability follow one another. The latter are defined by $m^{2} / 2<\kappa<m^{2} / 2+\varepsilon$. For any given $\varepsilon$ the maximum growth rate $\Gamma_{\max }=2 \varepsilon$ is a universal quantity for all modes which is independent of $m$ and is established at the coupling strengths $\kappa=\frac{1}{2}\left[m^{2}+\varepsilon\right]$. Note that for sufficiently large $\varepsilon$ the unstable regions of two adjacent $m$-modes may even overlap thus eliminating the stable region in between. This can be seen in fig.1p, where for a nonlinear energy $\varepsilon=2.0$ part of the regions of instability for the $m=1$ and $m=2$ modes overlap. Since the coupling $\kappa$ is a function of the trapping potential, and as such experimentally accessible, the instability of the modes can be used to selectively affect one or more $m$-modes of the rings. For example, at $\varepsilon=1.0$ each mode is "individually addressable" through an appropriate choice of $\kappa$ (see fig.1 1 ). At $\varepsilon=2.0$ and $\kappa=2.1$, on the other hand, both the $m=1$ and $m=2$ are unstable with respect to fluctuations.

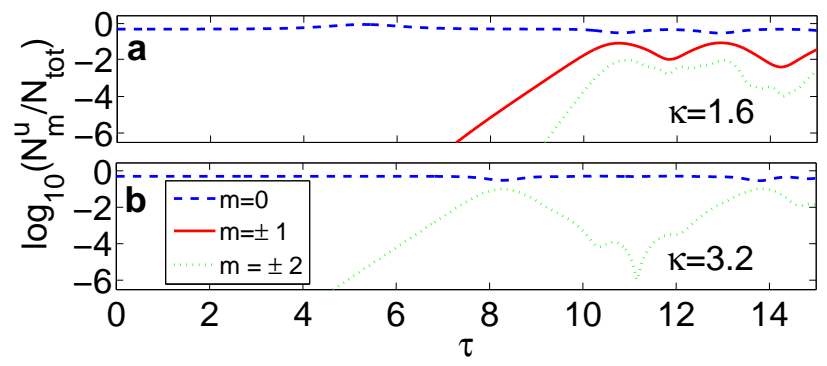

FIG. 2: (Color online) Evolution of the occupation number $N_{m}^{u}$ normalized to the total number of particles for the nonlinear energy $\varepsilon=2.0$. For $\kappa=1.6$ (panel a) we observe an exponential population increase in the $m= \pm 1$ modes. Population of the $m= \pm 2$ mode is also visible at later times but is suppressed by more than two orders of magnitude. For $\kappa=3.2$ (panel b), the accumulation in population of the $m= \pm 1$ modes is suppressed and only the exponential growth of the $m= \pm 2$ modes is visible.

The growth of the instable modes eventually leads to a break down of the linearized eqs.(5) due to the interaction between higher lying m-modes. We therefore return to eq.(3) and integrate them numerically. We choose $\alpha_{0}^{u / d}=\sqrt{N_{0}+\delta_{u / d}}$ as initial condition, i.e. almost equal number of atoms in the $m=0$ mode of both rings, whilst allowing for experimentally unavoidable particle number fluctuations of the order of $\delta_{u, d}=\mathcal{O}\left(\sqrt{N_{0}}\right)$. Adding these 
fluctuations has only minor influence on the numerical results. Following Saito et al. [9], we introduce a small seed in the lowest few angular momentum modes (up to $m= \pm 5$ ) with a magnitude of $10^{-4} \times \sqrt{N_{0}}$. Again, such a fluctuation is experimentally inevitable. We truncate the set of coupled eqs.(3) at the angular momentum mode $m= \pm 15$, well above the highest contributing mode. We verified the quality of the propagation by monitoring energy, norm, and angular momentum conservation. Since both annuli have a slight population difference the nonlinear energy is calculated according to $\varepsilon=\frac{\gamma N_{\text {tot }}}{4 \pi}$ with $N_{\text {tot }}=N_{u}+N_{d}$. An example of the numerical propagation can be seen in fig.2. We show the occupation $N_{m}^{u}$ for $m=0, \pm 1, \pm 2$ at the nonlinear energy $\varepsilon=2.0$ (see also fig.1 $\mathrm{b}$ ) for two different coupling strengths $\kappa$. For $\kappa=1.6$ only the $m= \pm 1$ modes are unstable. We observe for early times $(\tau<10)$ the predicted exponential increase in population with a rate of $\Gamma \approx 4$. At later times $\tau>9$ the population also in the $m= \pm 2$ modes increases slightly. This cannot be described by the linearized eqs.(5). However, in the time window considered here, the population of the $m= \pm 1$ modes is more than two orders of magnitude larger than one of the $m= \pm 2$ modes. In the lower panel we present the same plot for $\kappa=3.2$. Here we observe no population growth within the $m= \pm 1$ modes but $N_{ \pm 2}^{u}$ grows at a rate of $\Gamma=3.9$. This clearly demonstrates the possibility of a selective angular momentum mode excitation by tuning $\kappa$.

Let us now turn to the angular momentum of the two BECs. The $L_{z}$ expectation value of $\omega_{-}$branch is $\left\langle L_{z}^{u}\right\rangle_{-}^{m}=\left\langle L_{z}^{d}\right\rangle_{-}^{m}=0$ which implies an equal population of states with opposite $m$. Conversely, for modes of the branch $\omega_{+}$we find $\left\langle L_{z}^{u}\right\rangle_{+}=\left\langle L_{z}^{d}\right\rangle_{+}=\frac{m}{2} \frac{\sqrt{m^{4}+2 \varepsilon m^{2}}}{m^{2}+\varepsilon}$. However, according to eq.(66) the value of $\omega_{+}$is always real, which results in a growth rate of $\Gamma=0$. In fig. 3 we present the angular momentum per particle and the relative particle number difference between the coupled BECs. The nonlinear energy is again $\varepsilon=2.0$. The coupling is $\kappa=2.1$, where both the $m= \pm 1$ and the $m= \pm 2$ are unstable. Until $\tau=\tau_{\text {osc }} \approx 11$ we find very small oscillations of the relative particle difference which are of the order of $10^{-2}$. The decompositions into $m$ modes shows that this is due to small oscillations taking place between the $m=0$ modes of the two rings (see also fig. 2 for $\tau<6$ ). These are "ordinary" Josephson oscillations. Here no formation of currents, i.e $\left\langle L_{z}^{u / d}\right\rangle \neq 0$, takes place in either of the annuli. For $\tau>11$ the situation changes dramatically. We find particle oscillations up to $\left|N^{d}-N^{u}\right| / N_{\text {tot }}=0.4$ and a nonzero expectation values for $\left\langle L_{z}^{u / d}\right\rangle$. The time $\tau_{\text {osc }}$ of the onset of this regime depends on the magnitude of the initial seed $\alpha_{m}$. A rough estimate for $\tau_{\text {osc }}$ 


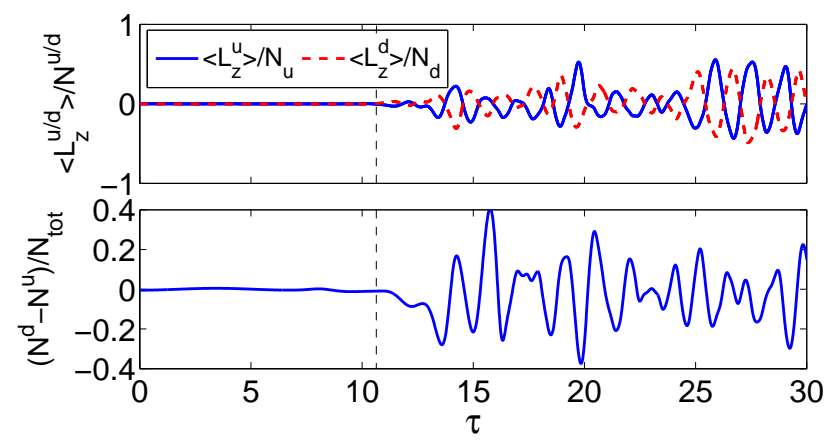

FIG. 3: (Color online) Angular momentum per particle in units of $\hbar$ and relative particle difference between the two annuli $(\varepsilon=2.0$ and $\kappa=2.1)$. For $\tau<11$ small oscillations in the particle difference in the order of $10^{-2}$ take place. At $\tau=\tau_{\text {osc }} \approx 11$ (dashed vertical line) we observe the onset of oscillations with bigger amplitude which are accompanied by angular momentum Josephson oscillations.

can be obtained from the equation $N_{0} \approx N_{m}=\left|\alpha_{m}(t=0)\right|^{2} \exp \left(\Gamma \tau_{\text {osc }}\right)$. These oscillations in $\left\langle L_{z}^{u / d}\right\rangle$ are due to the population of $\omega_{+}$modes caused by the nonlinear evolution of the system.

The oscillations spontaneously emerging for $\tau>\tau_{\text {osc }}$ are Josephson oscillations of the angular momentum. They break the chiral symmetry of the initial state's wave function where none of the rings carried a net angular momentum.

Finally, we turn to the experimental realizability of the "ordinary" and angular momentum Josephson oscillations. For ${ }^{87} \mathrm{Rb}$ and a ring radius of $\rho_{0}=1.2 \mu \mathrm{m}$ the energy scale given by the length of the ring evaluates to $E_{0} \approx k_{B} \times 2 n K$ and consequently we find a time scale of $\tau_{0} \approx 4 \mathrm{~ms}$. For a radial oscillator length of $a_{\rho}=0.3 \mu \mathrm{m}$ a nonlinear energy of $\varepsilon=2.0$ is achieved for a particle number $N_{0} \approx 50$ [16]. The experimental feasibility of building ring-shaped traps has been demonstrated recently [10, 11]. We therefore hope the results presented in this letter might stimulate further experiments.

The required smallness of the ring traps forbids direct in-situ imaging of the BECs. Therefore one has to employ time-of-flight (TOF) imaging. We consider an experiment where initially two annular BECs are created in two uncoupled ring trap from one single BEC. Subsequently the barrier in z-direction is lowered such that a certain coupling strength $\kappa$ is established. The system then evolves at constant $\kappa$ for a certain time $\tau_{\text {int }}$ after which the trap is switched off. The TOF image is then taken after free expansion of the cloud. 

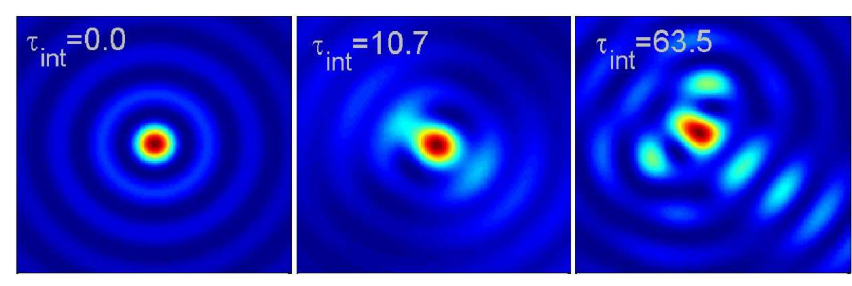

FIG. 4: (Color online) Simulated TOF images for several interaction times. The parameters correspond to those of the top panel of figure 2, The axis are in arbitrary units. For an interaction time of $\tau_{\text {int }}=0.0$ only the $m=0$ mode contributes to the momentum distribution yielding an image of the squared zero-th Bessel function. At $\tau_{\text {int }}=10.7$ the $m= \pm 1$ are occupied as well which gives rise to an angular modulation proportional to $\sin ^{2}(\phi)$ of the TOF image. For later times several angular momentum modes are occupied. We show an example for $\tau_{\text {int }}=63.5$. Here both annuli carry a net angular momentum.

Here we assume that only the wave function of one annulus is imaged, i.e. the atoms in the second annulus have to be removed [17]. The TOF method yields an image of the momentum distribution of the BEC [12, 13, 14]. This is equivalent to the squared modulus of the Fourier transform of the wave function $\Psi(\mathbf{r})$. Using the angular momentum mode decomposition $\alpha_{m}$ of the annulus which is to be probed and assuming that $a_{\rho}, a_{z} \ll R$ we obtain

$$
\Psi(\mathbf{k}) \propto \sum_{m=\text { even }}(-1)^{m / 2} J_{|m|}(k R) \alpha_{m} e^{i m \zeta}-\sum_{m=\text { odd }}(-1)^{(m-1) / 2} J_{|m|}(k R) \alpha_{m} e^{i m \zeta}
$$

with $J_{n}$ being the $n$-th Bessel function of the first kind, $k=\sqrt{k_{x}^{2}+k_{y}^{2}}$ and $\zeta=\arctan \left(k_{y} / k_{x}\right)$. Hence imaging $|\Psi(\mathbf{k})|^{2}$ allows the reconstruction of the $\alpha_{m}$ for a small number of contributing modes (for an example see fig.44). This allows an experimental study of the instability regions simply by a analyzing TOF images.

In summary, in a system consisting of two ground state BECs in coupled rings the occupation number of high angular momentum modes grows exponentially for well-defined coupling strengths. For small evolution times, a symmetric occupation of $\pm m$ modes takes place in each BEC accompanied by "ordinary" Josephson oscillations of the relative particle number. For later times, angular momentum Josephson oscillations spontaneously emerge. This novel type of Josephson oscillations breaks the initial chiral symmetry of the individual BECs. 
This research project has been supported by a Marie Curie Transfer of Knowledge Fellowship (IL) and a Marie Curie Excellence Grant (WK) of the European Communitys Sixth Framework Programme under the contract numbers MTKD-CT-2004-014496 and MEXTCT-2005-024854.

* igor@iesl.forth.gr

$\dagger$ wvk@iesl.forth.gr

[1] B. D. Josephson, Phys. Lett. 1, 251 (1962)

[2] J. C. Davis and R. E. Packard, Rev. Mod. Phys. 74, 741-773 (2002)

[3] A. Smerzi, S. Fantoni, S. Giovanazzi, and S. R. Shenoy, Phys. Rev. Lett. 79, 4950 (1997)

[4] M. Albiez et al., Phys. Rev. Lett. 95, 010402 (2005)

[5] G. J. Milburn, J. Corney, E. M. Wright and D. F. Walls, Phys. Rev. A 55, 4318 (1997)

[6] K. K. Das, M. D. Girardeau, and E. M. Wright, Phys. Rev. Lett. 89, 110402 (2002)

[7] I. Bouchoule, Eur. Phys. J. D 35, 147-154 (2005)

[8] C. J. Pethick and H. Smith, Bose-Einstein Condensation in Dilute Gases, Cambridge University Press (2002)

[9] H. Saito, Y. Kawaguchi and M. Ueda, Phys. Rev. Lett. 96, 065302 (2006)

[10] S. Gupta, K. W. Murch, K. L. Moore, T. P. Purdy, and D. M. Stamper-Kurn, Phys. Rev. Lett. 95, 143201 (2005)

[11] S. Hofferberth, I. Lesanovsky, B. Fischer, J. Verdu, and J. Schmiedmayer, Nature Physics 2, 710-716 (2006)

[12] M. Cozzini, B. Jackson and S. Stringari, Phys. Rev. A 73, 013603 (2006)

[13] I. Lesanovsky, S.Hofferberth, J.Schmiedmayer and P. Schmelcher, Phys. Rev. A 74, 033619 (2006)

[14] M. Modugno, C. Tozzo, F. Dalfovo, preprint:cond-mat/0605183

[15] Unless otherwise stated all quantities will be given in units of the scaled time $\tau_{0}=\frac{2 M R^{2}}{\hbar}$ and energy $E_{0}=\frac{\hbar^{2}}{2 M R^{2}}$

[16] These values have been calculated under the assumption that the states $\Phi\left(z \pm z_{0}\right)$ are harmonic oscillator ground states with an oscillator length $a_{z}=0.5 \mu \mathrm{m}$. The scattering length for ${ }^{87} \mathrm{Rb}$ was taken to be $5.2 \mathrm{~nm}$. 
[17] This can be achieved by first separating the rings and discarding one of them 